\title{
The Global Initiative for Asthma (GINA): 25 years later
}

\author{
Louis-Philippe Boulet ${ }^{1}$, Helen K. Reddel (1) ${ }^{2}$, Eric Bateman ${ }^{3}$, Søren Pedersen ${ }^{4}$, \\ J. Mark FitzGerald ${ }^{5}$ and Paul M. O'Byrne ${ }^{6,7}$
}

\author{
Affiliations: \\ ${ }^{1}$ Québec Heart and Lung Institute, Laval University, Québec City, QC, Canada. \\ ${ }^{2}$ Woolcock Institute of Medical Research, University of Sydney, Sydney, Australia. \\ ${ }^{3}$ Dept of Medicine, University of Cape Town, Cape Town, South Africa. \\ ${ }^{4}$ Kolding Hospital, Kolding, Denmark. \\ ${ }^{5}$ Institute for Heart and Lung Health, University of British Columbia, Vancouver, BC, Canada. \\ ${ }^{6}$ Firestone Institute of Respiratory Health, St Joseph's Healthcare, McMaster University, Hamilton, ON, \\ Canada. \\ ${ }^{7}$ Dept of Medicine, Michael G. DeGroote School of Medicine, McMaster University, Hamilton, ON, Canada.
}

\section{Correspondence:}

Louis-Philippe Boulet, Institut Universitaire de Cardiologie et de Pneumologie de Québec (IUCPQ), 2725, Chemin Sainte-Foy, Quebec, G1V 4G5, Canada.

E-mail: Ipboulet@amed.ulaval.ca

\section{@ERSpublications}

GINA, launched in 1993, produces an annual evidence-based report on the optimal management of asthma intended for global use, along with various resources, in addition to promoting initiatives to improve asthma care. bit.ly/2W3yDpU

Cite this article as: Boulet L-P, Reddel HK, Bateman E, et al. The Global Initiative for Asthma (GINA): 25 years later. Eur Respir J 2019; 54: 1900598 [https://doi.org/10.1183/13993003.00598-2019].

ABSTRACT The Global Initiative for Asthma (GINA) was launched in 1993 under the auspices of the National Heart, Lung, and Blood Institute, National Institutes of Health, USA, and the World Health Organization to produce a global strategy on asthma management and prevention. Now constituted as a non-profit entity, it continues to produce, on an annual basis, the most widely cited evidence-based report on the optimal management of asthma in both adults and children intended for global use. Although the GINA Report is often viewed and used as an asthma treatment guideline, it is designed to be a clinically oriented strategy document that supports the development of practice guidelines in different countries and regions.

Other GINA products, including the report's pocket guides, teaching slide kits and implementation tools, are also offered free of charge for public use. The GINA Scientific Committee comprises recognised international experts from primary, secondary and tertiary centres of care who are actively involved in both the care of patients and research in asthma. The GINA Assembly is a forum for exchange of scientific information and discussions on initiatives to improve asthma care in various countries, focusing on implementation strategies. GINA plays a role in shaping research on the diagnosis and treatment of asthma and informs the development of point of care practice guides and decision support tools. GINA supports the objectives of raising awareness of asthma and improving access to therapy and quality of care for asthmatic patients, in addition to presenting and promoting continuously updated evidence-based treatment approaches for global use. 


\section{Introduction}

At the end of the 1980s and early 1990s, a number of countries published guidelines on the diagnosis and management of asthma. These included Australia and New Zealand [1], Canada [2] and the UK [3]. The development of these documents was stimulated by concern about the marked increase in asthma prevalence in many countries, and a sharp rise in asthma deaths in the 1970s and 1980s [4]. In the USA, a National Asthma Education and Prevention Program (NAEPP) was initiated by the US National Heart, Lung, and Blood Institute (NHLBI) to address the growing problem of asthma in the USA, and an "Expert Panel Report on Diagnosis and Management of Asthma" was released in 1991 [5]. These publications changed common perceptions about asthma and its treatment by emphasising the role of inflammation in disease development, the need to obtain objective monitoring of lung function both for diagnosis and follow-up, and the need to establish partnerships between patients and healthcare providers and improve patient education. However, early documents focused on the health environment of the origin countries of the guidelines and did not have a global focus.

With the recognition that the increasing prevalence and morbidity of asthma was a global concern, an "International Consensus Conference on Asthma Management" was convened in 1991 in which representatives from the World Health Organization (WHO) and NHLBI participated [6]. A major outcome of the meeting was the recommendation to implement a programme to develop evidence-based recommendations for asthma care that could be applicable in all countries of the world. The NHLBI and the WHO agreed to implement this programme and formed a steering committee with the aim of preparing an "International Consensus Report" [6]. In 1993 the steering committee recommended the formation of a Global Initiative for Asthma (GINA) and identified additional experts to assist with the preparation of the document.

\section{GINA: the early years}

The initial GINA Report, published in 1995, was entitled "Global Strategy for Asthma Management and Prevention: NHLBI/WHO Workshop Report” [7]. The expert panel was chaired by Dr Albert Sheffer, with the invaluable support and assistance of Drs Suzanne Hurd (Scientific Director) and Claude Lenfant from the NHLBI (figure 1). The overall goal of the GINA Report is to improve the lives of people with asthma across the world. The main objectives of the GINA Report were then, and remain today, to increase awareness of asthma, to promote a shared understanding of the pathogenesis of asthma and its consequences, to promote evidence-based practices for its diagnosis and management, and to identify knowledge gaps to stimulate research. This should help reduce asthma morbidity and mortality by improving its management and promoting availability and accessibility of asthma therapy.

Companion documents developed by GINA included a pocket guide for health professionals, a patient guide, a guide for public health officials and a teaching slide set (table 1). Initially, these documents were printed and distributed through the NHLBI and multiple translations were made and distributed worldwide.
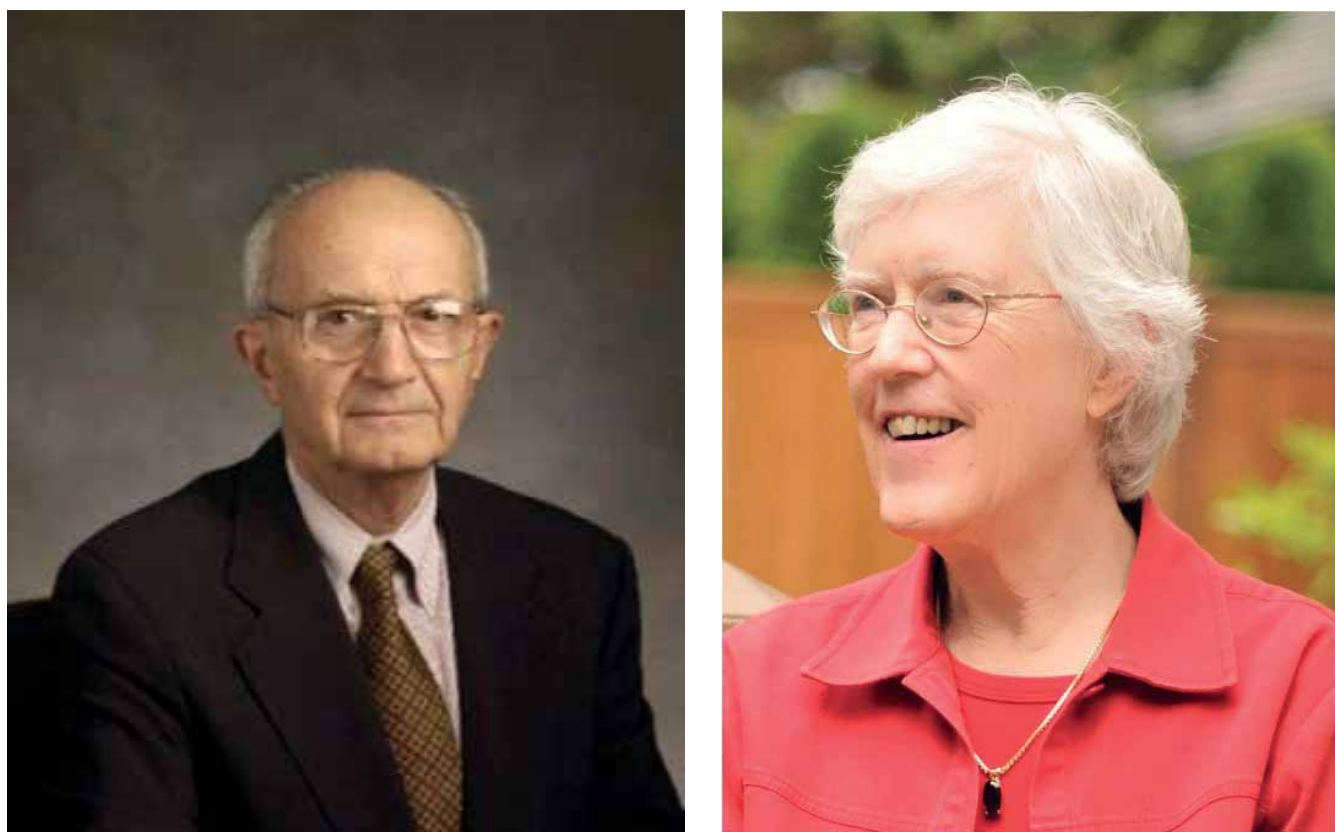

FIGURE 1 Dr Claude Lenfant and Dr Suzanne Hurd. 


\begin{tabular}{|c|c|}
\hline Product title & Revision schedule \\
\hline $\begin{array}{l}\text { GINA Report, Global Strategy for Asthma } \\
\text { Management and Prevention }\end{array}$ & Revised yearly \\
\hline $\begin{array}{l}\text { Pocket Guide for Asthma Management and } \\
\text { Prevention }\end{array}$ & Revised yearly; available in many languages \\
\hline GINA Teaching Slide Set & Revised yearly \\
\hline Implementation Toolbox & $\begin{array}{l}\text { Includes a frequently asked questions list and various } \\
\text { practice tools; will be continuously updated }\end{array}$ \\
\hline Patient Guide on Asthma Control & Under revision \\
\hline Videos and podcasts on main themes & Available on website; will be regularly updated \\
\hline
\end{tabular}

The initial work of GINA was supervised by an Executive Committee which met yearly, but in early 2000 a Science Committee was constituted to prepare the first major revision of the 1995 report, based on rapidly changing knowledge on asthma and its management. In addition, a mechanism was put in place to support more frequent review of scientific literature to ensure that important scientifically valid, clinically relevant emerging knowledge was included in the GINA Report in a timely manner. GINA's frequent review of the peer-reviewed literature informs annual updates (in addition to major revisions described below), and is a distinguishing feature of the approach used by GINA as compared to that used for reports and guidelines produced by other groups, often revised only at intervals of $\geqslant 5$ years.

Major revisions of the GINA Report that not only added new information but also reviewed the entire management of asthma in light of advances in our understanding of the disease were published in 2002 [8], 2006 [9] and again in 2014 [10]. Reports are posted on the GINA website (www.ginasthma.org), together with publications in peer-reviewed journals that describe the new or revised recommendations contained in each edition. In addition, annual updates are prepared and posted on the GINA website, usually in January or February each year.

Other important milestones in GINA's 25-year history are its registration in 2008 as a non-profit, tax-exempt organisation under the direction of a Board of Directors and, in 2014, the achievement of financial independence from health-industry-related companies, including pharma. All GINA activities are supported by the proceeds of sales or licensing of its products. Members of all GINA committees serve voluntarily, and receive no honoraria or expenses to attend the twice-yearly scientific review meetings, or for the time spent reviewing the literature and contributing substantively to the writing of the report. Representation from GINA is prominent in several global bodies, such as the Global Alliance against Chronic Respiratory Diseases of the WHO and the Federation of Respiratory Societies (FIRS).

Other initiatives during the first decade of GINA's existence were the creation of World Asthma Day, held annually on the first Tuesday of May, and the formation of a GINA Assembly comprising asthma experts from many countries. Working with these experts, several country-specific asthma management programmes have been initiated based on information presented in the GINA documents. World Asthma Day has proved to be an important tool for meeting the goals of raising awareness about the burden of asthma and dissemination of information presented in GINA documents.

\section{GINA structure and committees}

The structure of GINA includes the Board of Directors that, apart from constant communications through electronic means as for the other committees, meets three times a year; the Science Committee; the Dissemination and Implementation (D\&I) Committee; and the GINA Assembly (figure 2). The Science Committee comprises experts recognised internationally for their leadership in asthma research and clinical care, and membership is by invitation. Clinical expertise and scientific credentials in the field of asthma are a prerequisite, but representation from different continents, paediatrics and clinicians in primary care are important considerations.

The D\&I Committee focuses on the optimal dissemination and implementation of GINA recommendations, and the production of various implementation tools to assist this process. It recognises that implementation demands, where possible, evidence-based adaptation of generic recommendations to align with local practice conditions, resources and availability of drugs. Together with the Science Committee, the D\&I Committee screens the growing body of implementation science literature, and draws on successful examples of implementation in countries and regions to inform recommendations and provide resources for the Dissemination Toolkit. 


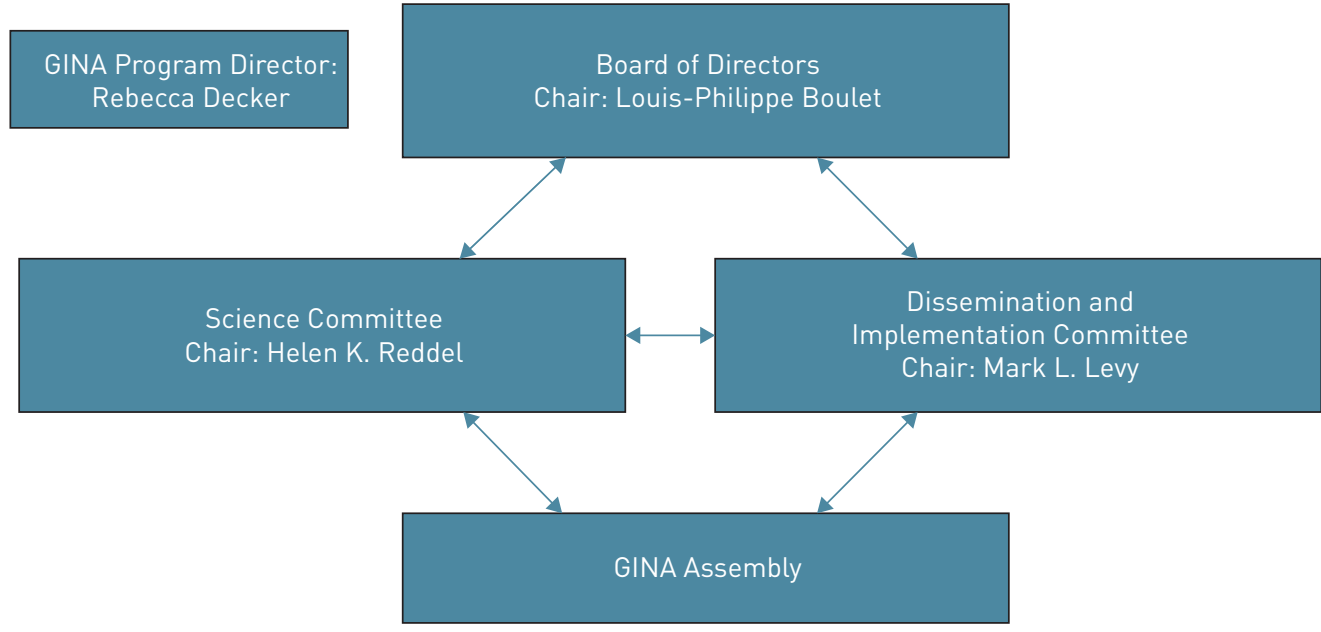

FIGURE 2 Global Initiative for Asthma (GINA) committees 2019.

The GINA Assembly, which meets yearly (in addition to contact through electronic communications), comprises clinicians and healthcare professionals involved in country efforts to promote GINA and improved asthma care. Members of the Assembly share experiences and insights on asthma implementation, and provide valuable feedback on GINA materials and their relevance to clinical practice and local conditions.

To a major extent, the success of GINA resulted from the immense contribution of Drs Claude Lenfant and Suzanne Hurd from its inception until their retirement in 2015. Claude and Suzanne worked tirelessly to coordinate and oversee all aspects of the organisation's activities. Both brought to GINA their exceptional experience gained during their long and distinguished service at the NHLBI, and they played a vital role not only through organisational and scientific leadership, but also through their inspiring commitment to improve asthma management worldwide. Their friendships and visits to many countries, and their influence in the field of respiratory medicine and public health, raised the profile of asthma care and established local country movements devoted to this cause. In January 2016, Ms Rebecca Decker took over as Global Program Director.

\section{Evolution of the GINA Report}

The GINA Report has evolved over time from an expert consensus statement to an evidence-based document, with recommendations based on analysis of the most current data, including meta-analyses, systematic reviews and other sources of information. Importantly, however, GINA has resolved and acknowledges that the main GINA Report does not attempt to conform to the relatively recently proposed rigorous methodologies for the development of guidelines, such as AGREE (Appraisal of Guidelines, Research and Evaluation) [11] and GRADE [12]. Several factors were considered in adopting this position. 1) In order to be globally relevant, the GINA Report must provide strategies that are evidence-based but these strategies require further consideration and adaptation before being included in local practice tools or guidelines. 2) With the GRADE approach to evaluation of evidence, the value that is placed on internal validity is higher than the value placed on external validity. Although GRADE allows evidence to be downgraded because of indirectness when not relevant to the intended context of use, the most robust evidence in international guidelines is based on research performed in developed rather than low-resource countries and settings. 3) Many, if not most, critical dilemmas in resource-poor settings are not considered in guidelines, nor is there evidence to support the best option. 4) The PICOT approach (Population, Intervention, Comparator, Outcomes, Timeframe) to guidelines informs the literature search process and helps readers identify the questions addressed by the guideline. Although relevant, this approach is not always helpful for clinicians in primary care, given that not all questions, and the format in which they are presented, are readily interpretable by primary care clinicians. 5) Preparing guidelines using systematic reviews of specific PICOT questions is time-consuming and expensive, and would result in less frequent updates. In addition the number of potential PICOT questions that would need to be addressed in a de novo revision of the current GINA Report would be in the hundreds. Moreover, the approach recommended by GRADE does not necessarily produce different recommendations when compared to more pragmatic approaches that rely on consensus of experts [13]. The GINA methodology ensures that literature is under regular review; evidence-based advances are more rapidly evaluated and, if relevant, are 
placed within the context of current recommendations. In reality GINA has now accumulated a 25-year repository of evidence-based recommendations that provides an agile framework for the integration of new and emerging evidence. However, the Science Committee adheres to a simpler but still widely used method of grading evidence, developed by the NHLBI.

In summary, for yearly reviews, after an initial screening of titles and abstracts for relevance, all members of the Committee receive a summary of citations and all abstracts. Each abstract is assigned to at least two non-conflicted Committee members, although all non-conflicted members are offered the opportunity to provide an opinion on all abstracts. Members evaluate the abstract and the full publication, answer four specific written questions, and indicate if the scientific data presented affect recommendations in the GINA Report. If so, the member is asked to specifically identify modifications that should be made. A detailed description of GINA methodology is available at https:/ginasthma.org/about-us/methodology/.

An extensive revision of the GINA Report occurred in 2014 [10]. In addition to sections on management of asthma in adults, adolescents and children over 6 years old, it contained a section on the management of children aged $<6$ years and, in collaboration with the Global Initiative for Chronic Obstructive Lung Disease (GOLD), an approach for the assessment and treatment of patients with features of both asthma and chronic obstructive pulmonary disease (COPD), labelled asthma-COPD overlap.

\section{Changes to and current content of the GINA Report}

The GINA Report provides a definition of asthma and recommendations on how to make the diagnosis and assess asthma control, and proposes non-pharmacological and pharmacological treatment aiming at controlling symptoms and minimising risks of exacerbations or other consequences of the disease. The report also includes information on guided asthma self-management education, identification and treatment of co-morbidities, and management of exacerbations. Finally, a chapter deals with the translation of GINA recommendation into current care. In previous iterations of the report, many pages were devoted to details about the physiopathology of the disease and the rationale for treatment. To make the document easier to read, this information has been transferred to an appendix to the report.

The definition of asthma has evolved since the first GINA Report. The current definition, dating from 2014, considers asthma to be mainly an airway inflammatory disease, but recognises the possibility that some patients may present with minimal inflammation. The definition states that: "Asthma is a heterogeneous disease, usually characterised by chronic airway inflammation. It is defined by the history of respiratory symptoms such as wheeze, shortness of breath, chest tightness and cough that vary over time and in intensity, together with variable expiratory airflow limitation”. This definition thus distinguishes asthma from conditions such as eosinophilic bronchitis that are characterised by airway inflammation but without excess variability of lung function; in contrast to asthma, eosinophilic bronchitis is not associated with severe exacerbations or risk of death.

GINA has always stressed the need for objective measures of variable airway obstruction to make a diagnosis, and proposes a process for weaning patients already on treatment off their medication if needed to confirm the diagnosis. A list of differential diagnoses to consider is provided.

A major change in the paradigm for asthma management has occurred since 2006, in that asthma management now focuses on optimising asthma control in both of its domains, i.e. improving current asthma symptom control and minimising future risk, particularly of severe exacerbations. It also aims to reduce loss of lung function and reduce side effects of the medications used. Importantly, GINA does not combine symptom control and exacerbation risk numerically, because they may be dissociated, e.g. asthma-related death can occur even in a patient with few previous symptoms [14]. Asthma severity is now mostly defined by the level of treatment needed to control the disease $[15,16]$, rather than, as previously, a complex algorithm of symptoms, lung function and reliever use, which did not take treatment requirements into consideration.

Treatment strategies are still proposed using a stepwise approach (five steps), according to the medication needed to achieve control and minimise future risk. However, at each step, the choice of treatment focuses on patient-centred decisions based on 1) appropriate assessment, including modifiable risk factors and phenotype where relevant; 2) adaptation of treatment; and 3) review of the response.

Non-pharmacological treatment is considered a priority and a list of measures is suggested, particularly smoking cessation, regular exercise, avoidance of relevant exposures, weight loss in obese patients and self-management education.

For pharmacological treatment, the list of available medications has greatly increased since the initial GINA Report. The need for the the early introduction of anti-inflammatory treatment has become evident from large-scale studies $[17,18]$. Until the 2019 revision, it was proposed that inhaled corticosteroids (ICS) were introduced to reduce the risk of exacerbations, even if a patient had infrequent asthma symptoms 
(e.g. asthma symptoms or need for short-acting $\beta_{2}$-agonists (SABA) more than twice a month, or nocturnal asthma symptoms once or more a month), or if they had one or more risk factors for exacerbations (e.g. low lung function, exacerbation requiring oral corticosteroids in the last year or has ever been in intensive care for asthma).

Another significant change has occurred more recently, with the promotion of better phenotyping of asthma, particularly severe asthma, using biomarkers whenever available. The aim is to improve assessment and treatment targeting [19,20]. Furthermore, with the advent of biologics, the options for treating step 5 (severe asthma) have increased and now include an anti-IgE monoclonal antibody (omalizumab), anti-interleukin (IL)-5 monoclonal antibodies (mepolizumab, reslizumab, benralizumab) and, more recently, an anti-IL-4 receptor/IL-13 antibody (dupilumab). These choices will likely expand in the next few years, with several new agents under investigation. A recent initiative by GINA was the development of a practical pocket guide for assessment and management of difficult-to-treat and severe asthma, with a clinical decision tree that incorporates insights from best practice information design [21].

The relevance of managing co-morbid conditions to ensure optimal asthma control has been increasingly stressed in successive versions. Some of these conditions can mimic asthma or contribute to symptoms (e.g. laryngeal dysfunction, anxiety disorders, upper airways disease) and others may reduce responsiveness to treatment (e.g. smoking and concurrent COPD).

Particular attention is stressed by GINA to the common causes of difficult-to-control asthma resulting from persistent care gaps, such as incorrect diagnosis, inadequate inhaler technique, untreated relevant co-morbidities, poor adherence or persistent exposure to allergens, tobacco smoke, indoor or outdoor air pollution, or medications such as beta-blockers or (in some patients) nonsteroidal anti-inflammatory drugs.

In the process of reviewing clinical needs, important evidence gaps appear. An example of this is how to treat patients with features of both asthma and COPD. Recognition that this "evidence-free zone" created considerable confusion, especially for those practicing in the primary care setting, prompted GINA and GOLD to jointly draft the first version of the asthma-COPD overlap document. A clearly stated aim of the document is to provide pragmatic treatment recommendations for primary care, because current asthma and COPD guidelines, based on selected patients, have opposite safety recommendations about the use of long-acting bronchodilators without ICS. The chapter was also written with the intention that it would stimulate further research, an objective that it has clearly achieved given that it triggered a surge in the number of research papers, many very critical of the term and of the approach. One alternative approach proposed was more detailed phenotyping and treatment of "treatable traits", amid impressions that GINA promotes a "one size fits all" approach. Precise diagnosis, including recognising and treating treatable factors is unquestionably desirable, and, in fact, has always been recommended in the GINA Report. Individualisation of asthma management has always been a GINA key recommendation and with the development of new targeted therapies for severe asthma, the need to phenotype asthma and consider specific biomarkers has been part of our approach. However, eliminating terms like asthma and defining airways diseases according to inflammatory markers alone requires further evaluation in longitudinal studies in multiple settings and for all age groups before this can be promoted as standard of care. Instead, GINA continues to promote personalised care according to patient characteristics, including, where available, inflammatory biomarkers, particularly for severe asthma. In order to fulfil its global mandate, a major switch in diagnostic terms currently being recommended for specialist use is considered premature.

\section{Recent changes in the recommendations for mild asthma therapy}

The 2019 GINA strategy report includes a major change in its philosophy of treatment of mild asthma. This is based on research over a number of years, but is especially a result of the recent publication of two GINA-initiated studies [22, 23]. For safety, GINA no longer recommends treatment with SABA alone. There is strong evidence that such use of SABA, although providing short-term relief of asthma symptoms, does not protect patients from severe exacerbations, and that regular or frequent use of SABAs increases the risk of exacerbations and, of even greater concern, death. GINA now recommends that all adults and adolescents with asthma should receive either symptom-driven (in mild asthma) or daily ICS-containing controller treatment to reduce their risk of serious exacerbations.

\section{Paediatric asthma}

Paediatric asthma was included in GINA from the beginning. Children constitute the highest proportion of the asthma population. Globally, paediatric asthma is mainly managed by non-specialists. Therefore, efforts have always been made to make the paediatric chapters in GINA easy to read and clinically oriented, with recommendations based on the best available evidence, as for the adult section. Flow diagrams for non-specialists on how to assess and treat exacerbations in children are available. 
The number of paediatricians on the GINA committees has varied over the years from four to six, with representation from Scandinavia, Southeast Asia, South and North America, Africa, Europe and China. The World Asthma Day always includes several paediatric asthma initiatives. Many paediatricians are also involved in the GINA Assembly.

The asthma management aims for children are similar to those for adults: to achieve good symptom control and minimise future risk. However, important differences are recognised. Assessing asthma control in children is based largely on symptoms, history of exacerbations and impairment of daily activities, including the use of validated scores developed for children 6-12 years old. The role of lung function tests is discussed. The treatment strategy is also based on a stepwise approach based on clinical evidence obtained in paediatric studies. By 1995, GINA had already recommended using ICS early in children with persistent asthma, very similar to what most guidelines now recommend in 2019.

One of the main concerns about using ICS in children has always been the potential risks of long-term side effects, such as influence on bone mineral density, growth, cataracts and adrenal suppression. Therefore, these risks are discussed in detail and summarised in easy-to-read text boxes. A similar approach is used to consider the choice of inhaler delivery systems for various age groups of children with a focus on those that are used correctly and which systems, when delivering ICS, are least likely to be associated with adverse effects.

Management of children aged $<6$ years with wheeze has always been challenging owing to the difficulty of obtaining objective measurements in these age groups. Both children with asthma and healthy children may present with cough, wheeze or heavy breathing with viral infections. Therefore, deciding whether a child should be given controller treatment often has to be based on the pattern of symptoms, including the frequency, severity and duration of the wheezing episodes and the temporal pattern of symptoms (only with viral colds or also in response to other triggers). A positive family history of allergic disorders or the presence of atopy or allergic sensitisation increases the likelihood of an asthma diagnosis.

The provided list of potential differential diagnoses in these age groups is important. A treatment trial with daily ICS, with follow-up scheduled after 2-3 months to review the response, may be useful. A repeated review is also important because the pattern of symptoms tends to change over time in a large proportion of these children. Over the years, efforts to identify different phenotypes in childhood asthma have failed to be clinically helpful with the diagnosis or choice of treatment, but as knowledge expands, phenotyping may improve assessment and treatment.

\section{Dissemination and implementation}

The GINA Report and its pocket guides have been translated into many languages, and have been very successfully disseminated during its 25-year history. Implementation of the recommendations has been a more challenging problem. Efforts to improve this have included 1) considering "intrinsic implementability" in writing the report, 2) making the report easier to read (with the help of addenda) and 3) the inclusion of many tables, algorithms and figures for clinical practice. Furthermore, a document suggesting how to implement GINA locally or nationally has been published and includes suggestions to improve GINA application of recommendations into current care [24]. Implementation tools have been developed and are now available on the website. They include templates for action plans, reviews, reminders and other useful documents.

Other initiatives proposed by GINA have included the Asthma Challenge, encouraging regions or countries to gather data on asthma hospitalisations and develop initiatives, based on GINA recommendations, to reduce these by at least $50 \%$. The World Asthma Day initiative has been very successful in increasing awareness and widely disseminating information about asthma.

In regard to new modes of communication, videos on key messages were produced and a series of podcasts is now available on the GINA website. Finally, self-assessment questionnaires and structured educational initiatives are under development, which could eventually lead to a certification programme.

\section{Recognising leaders on asthma: the Ambassadors Program}

The GINA Ambassador Program highlights individuals, worldwide, who have been involved in asthma care in their country, improving policy around asthma care, implementing GINA strategies in their community, developing asthma education materials, building multidisciplinary asthma care teams and organising World Asthma Day activities. The GINA Ambassadors Program recognises and celebrates the important efforts of these individuals in improving asthma outcomes in many regions of the world.

\section{Role of GINA in promoting research on asthma}

Promoting research on asthma is one of the GINA's main goals. Much research on asthma has been promoted by the GINA Report, but GINA has had an even more direct involvement in research through 


\section{Box 1 Evolution of the Global Initiative for Asthma (GINA) in the last 25 years}

1993: GINA is launched under the auspices of the National Heart, Lung and Blood Institute (NHLBI), National Institutes of Health (NIH), USA (represented by Dr Claude Lenfant), and the World Health Organization (WHO) (represented by Dr Nikolai Khaltaev). Dr Albert L. Sheffer was the chair of this expert panel.

1995: Publication of the first GINA Report: "Global Strategy for Asthma Management and Prevention: NHLBI/WHO Workshop Report".

2000: A Science Committee is constituted to prepare the first major revision of the 1995 report lreleased in 2002). Since 2002, the GINA Report has been revised annually.

2001: GINA initiates an annual World Asthma Day to raise awareness about the burden of asthma.

2002: The GINA Report states that it is reasonable to expect that in most patients with asthma, control of the disease can and should be achieved and maintained.

2004: A "Dissemination Committee" is formed (First Chair: Dr Wan-Cheng Tan). This Committee will a few years later become the "Dissemination and Implementation Committee" (First Chair: Dr Louis-Philippe Boulet).

2005: GINA recommends implementing an approach to asthma management based on asthma control, rather than asthma severity.

2008: Registration of GINA as a non-profit, tax-exempt organisation under the direction of a Board of Directors. Financial independence achieved in 2014.

2014: Major revision of the GINA Report, identifying the importance of assessing modifiable risk factors as well as symptom control, and incorporating practical flow-charts and tables to increase utility of the report for clinical practice.

2015: Dr Claude Lenfant and Dr Suzanne Hurd retire from GINA. Ms Rebecca Decker becomes the GINA Program Director.

2019: Major revision of the GINA Report. On demand short-acting $\beta_{2}$-agonists are no longer recommended as a sole asthma treatment, even for the mildest forms of asthma.

the recent studies on mild asthma proposed by GINA members to increase evidence for the best choice for the treatment of mild asthma $[22,23]$. In future reports, GINA aims to more systematically identify areas in which evidence gaps exist.

\section{Conclusion}

GINA has evolved over the last 25 years to become the global reference for asthma management (box 1). GINA does not compete with national asthma treatment guidelines, but aims to provide a global clinically oriented resource on optimal practice strategies to improve asthma outcomes. GINA Reports are "living documents", being updated annually and published on the web (www.ginasthma.org), as are other documents to support its implementation. Major changes in the management of asthma are expected, particularly in the management of severe and difficult-to-treat asthma. The GINA approach to evidence synthesis will ensure that all evidence-based and clinically validated developments that affect the management of asthma will be included in a timely way. However, of equal importance is a continued emphasis on improving methods to implement optimal asthma care, especially in poorly resourced settings. GINA is a voluntary association of health professionals whose focus is on improving the recognition and care of persons with asthma. It is also a resource for and partner to professional societies and global movements that have similar agendas. Over 25 years, GINA has become a recognised brand, chiefly because of its tried and tested dual approach of continuous knowledge synthesis and evidence-based implementation, but also because of the dedicated contribution of clinicians and other health professionals from numerous countries in seeking better ways to promote GINA strategies into asthma care.

Conflict of interest: L-P. Boulet reports the following: research grants for participation in multicentre studies from AstraZeneca, Boston Scientific, GlaxoSmithKline, Hoffman La Roche, Novartis, Ono Pharma, Sanofi and Takeda; support for research projects introduced by the investigator from AstraZeneca, Boehringer Ingelheim, GlaxoSmithKline, Merck and Takeda; consulting fees and advisory board membership for AstraZeneca, Novartis and Methapharm; royalties as co-author of "Up-To-Date" (occupational asthma); non-profit grants for the production of educational materials from AstraZeneca, Boehringer Ingelheim, GlaxoSmithKline, Merck Frosst and Novartis; conference fees from AstraZeneca, GlaxoSmithKline, Merck and Novartis; and support for participation in conferences and meetings from Novartis and Takeda. He is the past president and a member of the Canadian Thoracic Society Respiratory Guidelines Committee and Chair of the Board of Directors of the Global Initiative for Asthma (GINA). H. Reddel reports grants and personal fees from AstraZeneca and Novartis; grants, personal fees and non-financial support from GlaxoSmithKline; and personal fees from Merck, Teva, Mundipharma and Boehringer Ingelheim; outside the submitted work. She is Chair of the GINA Scientific Committee. E. Bateman is a member of the Science Committee and Board of GINA. He reports personal fees from Novartis, Cipla, Vectura, Menarini, ALK, ICON, Sanofi Regeneron, Boehringer Ingelheim and AstraZeneca, and grants from GlaxoSmithKline, Hoffmann le Roche, Sanofi-Aventis, Novartis, 
AstraZeneca and Teva, outside the submitted work. S. Pedersen reports personal fees from AstraZeneca, ALK and Thermo Fisher, outside the submitted work. J.M. FitzGerald reports personal fees for advisory board membership from GSK, AstraZeneca, Boehringer Ingelheim, Novartis, Pfizer, Sanofi-Regeneron and Theravance, peer-reviewed funding from CIHR, AllerGen and BC Lung Association, research funding from GSK, AstraZeneca, Amgen, Sanofi-Regeneron and Novarits, all paid directly to UBC; and is a member of the steering committee for the International Severe Asthma Registry and PI for the Canadian Severe Asthma Registry, and a member of the GINA Science and Executive Committees. P.M. O'Byrne has received consultancy fees from AstraZeneca, GSK and Chiesi; has received grants/patents pending from AstraZeneca, Genetech and Novartis; and is a board member for the Joint Oversight Board for LABA safety study.

\section{References}

1 Woolcock A, Rubinfeld AR, Seale JP, et al. Thoracic Society of Australia and New Zealand. Asthma management plan, 1989. Med J Aust 1989; 151: 650-653.

2 Hargreave FE, Dolovich J, Newhouse MT. The assessment and treatment of asthma: a conference report. J Allergy Clin Immunol 1990; 85: 1098-1111.

3 Guidelines for Management of Asthma in Adults: I--Chronic Persistent Asthma. Statement by the British Thoracic Society, Research Unit of the Royal College of Physicians of London, King's Fund Centre, National Asthma Campaign. BMJ 1990; 301: 651-653.

4 Woolcock AJ. Worldwide trends in asthma morbidity and mortality. Explanation of trends. Bull Int Union Tuberc Lung Dis 1991; 66: 85-89.

5 National Asthma Education and Prevention Program. Expert Panel Report: Guidelines for the Diagnosis and Management of Asthma. Publication No. 91-3642. Bethesda, National Institutes of Health, 1991.

6 International Consensus Report on Diagnosis and Treatment of Asthma. Publication No. 92-3091. Bethesda, National Institutes of Health, 1992.

7 Global Initiative for Asthma. Global Strategy for Asthma Management and Prevention. NHLBI/WHO Workshop Report. Publication No. 95-3659. Bethesda, National Institutes of Health National Heart, Lung, and Blood Institute, 1995.

8 Global Initiative for Asthma. NHLBI/WHO Workshop Report: Global Strategy for Asthma Management and Prevention. Publication No. 02-3659. Bethesda, National Institutes of Health National Heart, Lung, and Blood Institute, 2002

9 Bateman ED, Hurd SS, Barnes PJ, et al. Global strategy for asthma management and prevention: GINA executive summary. Eur Respir J 2008; 31: 143-178.

10 Reddel HK, Bateman ED, Becker A, et al. A summary of the new GINA strategy: a roadmap to asthma control. Eur Respir J 2015; 46: 622-639.

11 AGREE Collaboration. Development and validation of an international appraisal instrument for assessing the quality of clinical practice guidelines: the AGREE project. Qual Saf Health Care 2003; 12: 18-23.

12 Schunemann HJ, Oxman AD, Brozek J, et al. Grading quality of evidence and strength of recommendations for diagnostic tests and strategies. BMJ 2008; 336: 1106-1110.

13 Schoenberg NC, Barker AF, Bernardo J, et al. A comparative analysis of pulmonary and critical care medicine guideline development methodologies. Am J Respir Crit Care Med 2017; 196: 621-627.

14 Dusser D, Montani D, Chanez P, et al. Mild asthma: an expert review on epidemiology, clinical characteristics and treatment recommendations. Allergy 2007; 62: 591-604.

15 Cockcroft DW, Swystun VA. Asthma control versus asthma severity. J Allergy Clin Immunol 1996; 98: 1016-1018.

16 Taylor DR, Bateman ED, Boulet LP, et al. A new perspective on concepts of asthma severity and control. Eur Respir J 2008; 32: 545-554.

17 Pauwels RA, Pedersen S, Busse WW, et al. Early intervention with budesonide in mild persistent asthma: a randomised, double-blind trial. Lancet 2003; 361: 1071-1076.

18 Reddel HK, Busse WW, Pedersen S, et al. Should recommendations about starting inhaled corticosteroid treatment for mild asthma be based on symptom frequency: a post-hoc efficacy analysis of the START study. Lancet 2017; 389: 157-166.

19 Chung KF. Precision medicine in asthma: linking phenotypes to targeted treatments. Curr Opin Pulm Med 2018; 24: 4-10.

20 Carr TF, Kraft M. Use of biomarkers to identify phenotypes and endotypes of severe asthma. Ann Allergy Asthma Immunol 2018; 121: 414-420.

21 Global Initiative for Asthma. Difficult-to-Treat and Severe Asthma in Adolescent and Adult Patients - Diagnosis and Management. A GINA Pocket Guide for Health Professionals. Fontana, Global Initiative for Asthma, 2019.

22 O’Byrne PM, FitzGerald JM, Bateman ED, et al. Inhaled combined budesonide-formoterol as needed in mild asthma. N Engl J Med 2018; 378: 1865-1876.

23 Bateman ED, Reddel HK, O'Byrne PM, et al. As-needed budesonide-formoterol versus maintenance budesonide in mild asthma. N Engl J Med 2018; 378: 1877-1887.

24 Boulet LP, FitzGerald JM, Levy ML, et al. A guide to the translation of the Global Initiative for Asthma (GINA) strategy into improved care. Eur Respir J 2012; 39: 1220-1229. 